\title{
On the stability of gas bubbles rising in an inviscid fluid
}

\author{
By D. I. MEIRON \\ Applied Mathematies, California Institute of Technology, Pasadena, CA 91125, USA
}

(Received 14 December 1987 and in revised form 12 May 1988)

The stability to three dimensional disturbances of bubbles rising rectilinearly in an inviscid fluid is studied numerically. It is found, in contrast with earlier work, that the interaction of hydrodynamic pressure forces and surface tension does not lead to linear instability of the bubble path.

\section{Introduction}

An easily observed example of the onset of instability is the spiralling or zig-zag motion of gas bubbles rising in a pure fluid. For bubbles rising freely in an infinite media, it is possible to identify (Clift, Grace \& Weber 1978; Harper 1972; Rosenberg 1950) three important dimensionless parameters which may be used to classify the motion. These are the Reynolds number,

$$
R e=\frac{2 \rho r_{\mathrm{e}} U}{\mu},
$$

the Eötvös number,

$$
E o=\frac{4 g \Delta \rho r_{\mathrm{e}}^{2}}{\sigma}
$$

and the Morton number,

$$
M=g \mu^{4} \frac{\Delta \rho}{\rho^{2} \sigma^{3}} .
$$

Here $\rho$ is the fluid density, $r_{\mathrm{e}}$ is the equivalent radius of the bubble which may be obtained directly from the bubble volume, $U$ is a characteristic velocity, $\mu$ is the dynamic viscosity, $g$ is the acceleration due to gravity, and $\sigma$ is the coefficient of surface tension. $\Delta \rho$ is the difference in densities between the fluid exterior to a droplet and the fluid interior. For bubbles $\Delta \rho=\rho$. The last of these parameters, the Morton number, is strictly a function of fluid properties and may be used to classify the type of fluid under consideration.

Pure fluids of low viscosity possess very low values of $M\left(\approx 10^{-9}\right)$ and it is for these fluids that spiralling is observed. The typical Reynolds number for the phenomenon is $R e=200$ to 1000 and the Eötvös number is in the range $1<E o<10$.

It is now commonly accepted that the spiralling motions are related to wake instability. However, until the numerical calculations of Ryskin \& Leal $(1984 a, b)$ it was unclear whether flow about a bubble free surface could generate a closed separation bubble. Ryskin \& Leal have presented numerical solutions of the steady axisymmetric Navier-Stokes equations which show a definite wake structure at moderate Reynolds numbers. While a stability analysis of these flows has yet to be 
performed, it is clear that an important assumption for the wake shedding hypothesis, i.e. the existence of a wake, is valid.

An alternative explanation of the spiralling phenomenon was proposed by Hartunian \& Sears (1957). They performed an experimental study in order to test a conjecture of von Kármán that the crucial dimensionless parameter in determining the onset of spiralling was the Weber number. The Weber number, given by $W e=$ $U\left(\rho r_{\mathrm{e}} / \sigma\right)^{\frac{1}{2}}$ may be computed from the Reynolds number, Eötvös number and Morton number and is a measure of the ratio of inertial forces relative to surface tension. The experiments showed the onset of instability at a critical Reynolds number of 200 for the fluids of higher viscosity (larger $M$ ) while for fluids of low $M$ the criterion was a critical Weber number of roughly 1.3. This led to the conclusion that for fluids of very low $M$ spiralling may be the result of an inviscid unstable interaction between surface tension and pressure.

Hartunian \& Sears (1957) then presented a stability analysis of the inviscid flow about a closed free surface taking into account the effects of surface tension. In order to simplify the analysis they initially assumed the rising bubble was spherical even for $W e>0$ and linearized the equations of motion about the steady state corresponding to a spherical bubble. This is not consistent since the inviscid flow deforms the free surface, and further calculations were made in an attempt to incorporate the deformation of the bubble during steady rise. All calculations revealed an instability of the centroid with those attempting to correct for deformation indicating a critical Weber number in close agreement with experiment.

In this paper we have repeated this analysis but, using a numerical approach, we have incorporated in a self-consistent manner the deformation of the bubble. Our results show no instability. In $\$ 2$ we state the problem in mathematical terms and describe the calculation of the steady states. A previous calculation of these steady solutions had been given by Miksis, Vanden-Broeck \& Keller (1981). In $\S 3$ the results of the stability analysis are presented and reasons are given for the discrepancy between our work and that of Hartunian \& Sears. Some conclusions are presented in $\$ 4$.

\section{Calculation of steadily rising inviscid bubbles}

We consider the motion of an incompressible fluid of small viscosity produced by a steady rising gas bubble. Viscosity and gravity are both neglected. In reality, the velocity of the bubble is determined by the balance between the small viscous and gravitational effects. The assumption being explored here is that, once these effects determine the rectilinear velocity, the shape of the bubble and the stability of the flow are governed by the balance between inviscid pressure forces and surface tension. We therefore assume the flow is irrotational. An alternative inviscid formulation which attempts to incorporate viscosity in an approximate way is given by Miksis, Vanden-Broeck \& Keller (1982). The velocity potential $\Phi$ satisfies Laplace's equation, and its evolution is governed by the Bernoulli equation evaluated at the bubble surface:

$$
\rho\left(\frac{\partial \Phi}{\partial t}+\frac{1}{2}|\nabla \Phi|^{2}\right)-\sigma\left(\frac{1}{R_{1}}+\frac{1}{R_{2}}\right)+p_{\mathrm{i}}=0 .
$$

Here $\rho$ is the density of the fluid, $\sigma$ is the coefficient of surface tension, $R_{1}, R_{2}$ are the principal radii of curvature of the bubble surface and $p_{1}$ is the interior pressure of the 
bubble. The fluid is assumed to be at rest at infinity and the (constant) pressure there is taken to be zero. In spherical polar coordinates $(r, \theta, \phi)(2.1)$ becomes

$$
\rho \frac{\partial \Phi}{\partial t}+\frac{1}{2} \rho\left[\left(\frac{\partial \Phi}{\partial r}\right)^{2}+\frac{1}{R^{2}}\left(\frac{\partial \Phi}{\partial \phi}\right)^{2}+\frac{1}{R^{2} \sin ^{2}(\theta)}\left(\frac{\partial \Phi}{\partial \phi}\right)^{2}\right]-\sigma\left(\frac{1}{R_{1}}+\frac{1}{R_{2}}\right)+p_{i}=0 .
$$

The bubble radius $R(\theta, \phi, t)$ obeys the kinematic condition

$$
\frac{\partial R}{\partial t}+\frac{1}{R^{2}} \frac{\partial R}{\partial \theta} \frac{\partial \Phi}{\partial \theta}+\frac{1}{R^{2} \sin ^{2}(\theta)} \frac{\partial R}{\partial \phi} \frac{\partial \Phi}{\partial \phi}=\frac{\partial \Phi}{\partial r}
$$

where all partial derivatives are evaluated on the bubble surface $r=R(\theta, \phi, t)$.

We consider solutions which represent steady flow caused by rectilinear motion of the bubble with constant velocity $U$. In this case we may rewrite the equations of motion in a frame translating with the bubble. We also restrict our attention to solutions which are axisymmetric about the direction of rise which we take as the $z$ axis. The potential $\Phi$ can be written

$$
\Phi=-U z+G(r, \theta)=-U r \cos \theta+G(r, \theta) .
$$

The equations of motion become

$$
\nabla^{2} G(r, \theta)=0
$$

exterior to the bubble with the conditions

$$
\begin{gathered}
\left(-U \cos \theta+G_{r}\right)-\frac{R_{\theta}}{R^{2}}\left(U R \sin \theta+G_{\theta}\right)=0 \\
\frac{1}{2}\left[\left(-U \cos \theta+G_{r}\right)^{2}+\frac{1}{R^{2}}\left(U R \sin \theta+G_{\theta}\right)^{2}\right] \\
-\frac{\sigma}{\rho}\left[\frac{R^{2}+2 R_{\theta}^{2}-R R_{\theta \theta}+\left(R^{2}+R_{\theta}^{2}\right)\left(1-\left(R_{\theta} / R\right) \cot \theta\right)}{\left(R^{2}+R_{\theta}^{2}\right)^{\frac{3}{2}}}\right]+\frac{p_{\mathrm{i}}}{\rho}=\frac{1}{2 U^{2}}
\end{gathered}
$$

imposed on the deformed surface. The subscripts denote differentiation with respect to the indicated variable.

For a given velocity $U$, equations (2.4-2.7) comprise a nonlinear boundary-value problem for the unknowns $R, G$. and $p_{\mathbf{i}}$. In terms of the dimensionless variables

$$
R^{\prime}=\frac{R}{r_{\mathrm{e}}}, \quad \Phi^{\prime}=\frac{\Phi}{U r_{\mathrm{e}}}, \quad p_{\mathrm{i}}^{\prime}=\frac{p_{\mathrm{i}} r_{\mathrm{i}}}{\sigma}
$$

the Bernoulli equation (2.7) becomes

$$
\frac{1}{2} W e^{2}\left|\nabla^{\prime} \Phi^{\prime}\right|^{2}-\left(\frac{1}{R_{1}^{\prime}}+\frac{1}{R_{2}^{\prime}}\right)=-p_{1}^{\prime}+\frac{1}{2} W e^{2}
$$

where $W e$, the Weber number, is defined in $\S 1$ and $r_{\mathrm{e}}$ is the equivalent radius of the bubble defined in terms of the bubble volume $V$ :

$$
V=\frac{4}{3} \pi r_{\mathrm{e}}^{3}
$$

The dimensionless form of the Bernoulli equation suggests that we must specify the Weber number in order to pose uniquely the solution to (2.4)-(2.7). Thus in addition to the bubble velocity, fluid density and coefficient of surface tension we must also 
specify the equivalent radius of the bubble. This is done through the volume condition

$$
\int_{0}^{\pi} R^{3}(\theta) \mathrm{d}(\cos \theta)=2 r_{\mathrm{e}}^{3}
$$

Finally in order to fix properly the origin of the spherical coordinate system we also specify the location of the bubble centroid:

$$
\int_{0}^{\pi} R^{4}(\theta) \cos \theta \mathrm{d}(\cos \theta)=0 .
$$

Equations (2.5)-(2.7), (2.10), (2.11) possess isolated solutions and can be used now to determine $p_{\mathrm{i}}, R(\theta)$, and $G(r, \theta)$ in terms of $U, \rho, \sigma$, and $r_{\mathrm{e}}$.

Solutions of these equations have been obtained numerically by Miksis et al. (1981) who utilized a boundary integral formulation of Laplace's equation. This results in a nonlinear integro-differential equation for the bubble surface and potential. We chose instead to represent the solution as a truncated series in Legendre polynomials :

$$
\begin{gathered}
R(\theta)=\sum_{n=0}^{N} A_{n} P_{n}(\cos \theta) \\
\Phi(r, \theta)=-U r \cos \theta+\sum_{n=0}^{N} B_{n} \frac{P_{n}(\cos \theta)}{r^{n+1}} .
\end{gathered}
$$

Since the volume is fixed there can be no source term in (2.13) and hence

$$
B_{0}=0 \text {. }
$$

The method of collocation is used to generate a nonlinear system of equations for the coefficients $A_{n}, B_{n}$, which is solved by Newton's method. Since our basis functions are Legendre polynomials a natural choice for the collocation points are the roots of $P_{N+1}(\theta)$ given by

$$
\theta_{i}: P_{N+1}\left(\cos \theta_{i}\right)=0 \quad(i=1, \ldots, N+1) .
$$

Evaluating the equations $(2.6,2.7)$ at the $N+1$ selected points yields $2 N+2$ equations. Along with the constraints $(2.10),(2.11),(2.14)$ there are $2 N+5$ equations for the $2 N+3$ unknowns $A_{n}, B_{n},(n=0, \ldots, N)$ and $p_{\mathrm{i}} \dagger$

This discrepancy between the number of equations and unknowns arises because the potential must obey integral constraints when the conditions (2.10) and (2.11) are satisfied. The conservation of volume implies that the system of equations $(2.12-2.14)$ will automatically satisfy

$$
\int \frac{\partial \Phi}{\partial n} \mathrm{~d} S=0 .
$$

Therefore the values of the potential at the collocation points are not independent and satisfy the discretized form of condition (2.16). As a result, the use of collocation on equations (2.6-2.7) would lead to a dependent system of equations. However the

$\uparrow$ The justification of the approach described above is not at all rigorous and is based on physical arguments for the existence of solutions to (2.17) with $B_{0}=0$. There are alternative methods for isolating unique solutions such as the choice of different collocation points $\theta_{i}$ or the use of a leastsquares approach. 
method of collocation has the advantage that it is much easier to implement numerically. In order to retain this advantage we use the method of inflation (akin to the method of undetermined multipliers) devised by Chen \& Saffman (1980) and rewrite the kinematic condition in the form

$$
\left(U \cos \theta-G_{r}\right)+\frac{R_{\theta}}{R^{2}}\left(U R \sin \theta+G_{\theta}\right)+f_{1}(\theta)\left[\int_{0}^{\pi} R^{4}\left(\theta^{\prime}\right) \cos \theta^{\prime} \mathrm{d}\left(\cos \theta^{\prime}\right)\right]+f_{2}(\theta) B_{0}=0
$$

where $f_{1}, f_{2}$ are arbitrary functions of $\theta$. We now retain (2.10) and drop (2.11) and (2.14). The number of equations, (2.6), (2.10), (2.17), now matches the number of unknowns. The arbitrary functions $f_{1}(\theta), f_{2}(\theta)$ are chosen so that the resulting Jacobian of the system is invertible. This artifice effectively folds in the extra equations and isolates the solutions obeying (2.11) and (2.14).

Note that our formulation is not restricted to bubbles which are symmetric about the plane $\theta=\frac{1}{2} \pi$. Previous work has examined only those solutions which are fore and aft symmetric. The existence of asymmetric solutions is thus an open question. If attention is restricted to symmetric solutions then the mismatch in the counting of equations and unknowns for the collocation method does not occur. The various constraints are satisfied automatically.

As the velocity $U$ is increased the bubble surface distorts away from its spherical shape and becomes increasingly oblate. Because of this there exists a critical value of the velocity past which (2.13) is no longer a convergent series for the potential everywhere on the bubble surface. The representation for the radius will always be convergent provided the surface is single valued and smooth. Thus the formulation described above will only be useful in calculating the flow about moderately distorted bubbles. Numerical experiments show that the representation (2.13) fails to converge when the ratio of the lengths of the semimajor axis to semiminor axes or aspect ratio exceeds 1.4 . In order to continue past this point we recast the problem in oblate spheroidal coordinates. These are defined by

$$
\left.\begin{array}{l}
x=d \cosh \mu \sin \xi \cos \zeta \\
y=d \cosh \mu \sin \xi \sin \zeta \\
z=d \sinh \mu \cos \xi
\end{array}\right\}
$$

The surfaces $\mu=$ constant are the family of oblate spheroids

$$
\frac{x^{2}}{d^{2} \cosh ^{2} \mu}+\frac{y^{2}}{d^{2} \cosh ^{2} \mu}+\frac{z^{2}}{d^{2} \sinh ^{2} \mu}=1
$$

The Laplace equation can be solved by separation of variables in this coordinate system. The coordinate $\mu$ and the potential $\Phi$ are now represented by the series

$$
\begin{gathered}
\mu(\xi)=\sum_{n=0}^{N} C_{n} P_{n}(\cos \xi) \\
\Phi(\mu, \xi)=-U d \sinh \mu \cos \xi+\sum_{n=0}^{N} D_{n} Q_{n}(\sinh \mu) P_{n}(\cos \xi),
\end{gathered}
$$


where the $Q_{n}$ are the irregular Legendre functions of imaginary argument suitably normalized so that the coefficients $D_{n}$ are real. The $Q_{n}$ are defined through the relations

$$
\left.\begin{array}{l}
Q_{0}=\tan ^{-1}\left(\frac{1}{\sinh \mu}\right), \\
Q_{1}=1-(\sinh \mu) \tan ^{-1}\left(\frac{1}{\sinh \mu}\right), \\
Q_{n}=-\left(\frac{2 n-1}{n}\right) \sinh \mu Q_{n-1}+\left(\frac{n-1}{n}\right) Q_{n-2}
\end{array}\right\}
$$

As long as $\sinh \mu<0.2$ formula (2.22) may be reliably used to compute the $Q_{n}$ numerically. However for $\sinh \mu>0.2$ the forward recursion is highly unstable. For these values we set $Q_{M}=0$ for some sufficiently large $M$ and recurse backwards. The correct values are then recovered by normalizing with respect to the known values of $Q_{0}$.

Provided the bubbles are sufficiently close in shape to an oblate spheroid, highly deformed surfaces may be computed by this method. The parameter $d$ in (2.18) fixes the size of the ellipsoid used to approximate the bubble and is determined through an extra condition which effectively fits an ellipsoid to the bubble surface. There are several ways to accomplish this. The one used here is to fit an ellipsoid at the points $\xi=0$ and $\xi=\frac{1}{2} \pi$ :

$$
\mu(0)=\mu\left(\frac{1}{2} \pi\right) .
$$

We emphasize that this condition only determines an appropriate value for the parameter $d$. Deviations from a purely ellipsoidal shape are then accounted for by the $\xi$-dependent terms in the series representation $(2.20)$.

Using equations (2.7), (2.10), (2.17) in spheroidal coordinates we solve this system by collocation and Newton's method. All calculations reported here were performed on a VAX 11/750 computer using 64 bit arithmetic.

The accuracy of the algorithms developed above was checked in several ways. For bubbles with small rise velocities it is possible to develop the solution using regular perturbation theory. The expansion parameter is the Weber number defined in (2.9). The leading-order terms to $O\left(W e^{2}\right)$ are

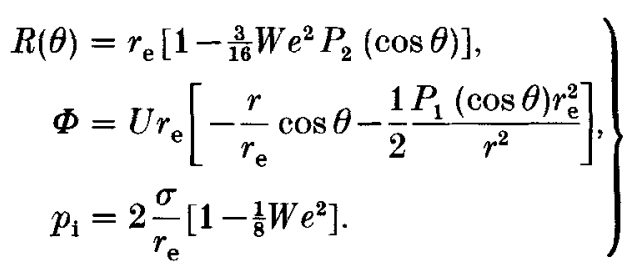

This solution was used to check the algorithm based on spherical harmonics for small values of $W e$ and excellent agreement was obtained. For larger values of $W e$ computations were performed using $N=20,40,60$, and 80 collocation points to assure convergence. As discussed previously the collocation method based on spherical harmonics ceases to provide convergent results at an aspect ratio $R_{\max } / R_{\min }=1.4$. At this point it became necessary to use the formulation based on oblate harmonics. Solutions were generated for $1.2<R_{\max } / R_{\min }<1.4$ and compared with the convergent results of the spherical harmonic formulation. It proved impossible to generate solutions for very low values of $W e$ since the sphere corresponds to a singular limit $(\mu \rightarrow \infty, d \rightarrow 0)$ in oblate spheroidal coordinates.

In addition to consistency checks made by increasing the number of collocation 


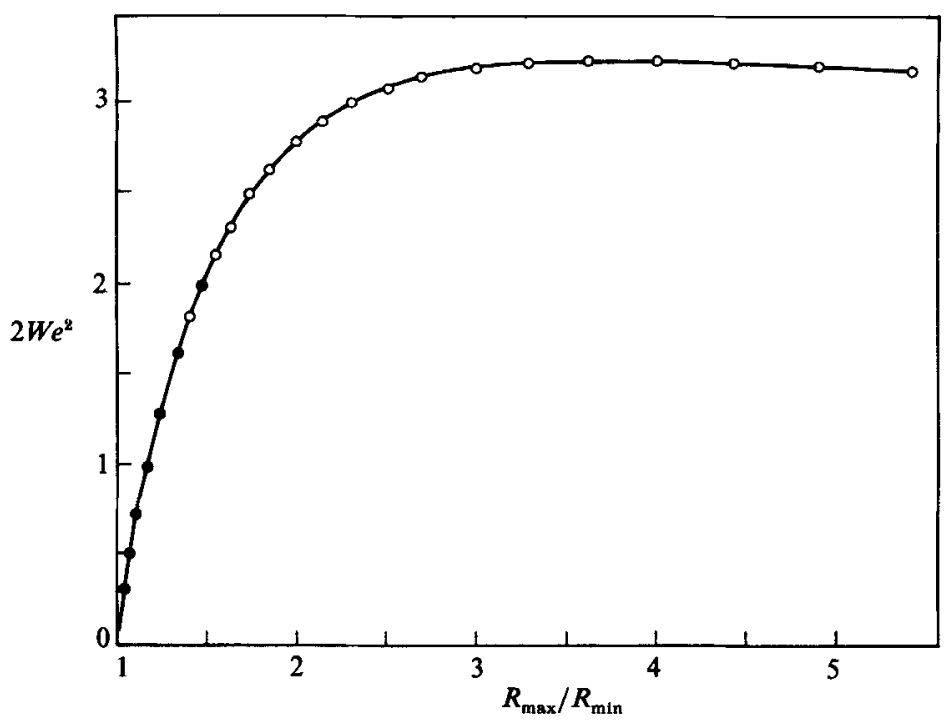

Figure 1. A plot of the quantity $\left(2 W e^{2}\right)$ where $W e$, the Weber number, is defined in $\S 1$ versus the deformation of the bubble as represented by the aspect ratio of the bubble $\left(R_{\max }=R\left(\theta=\frac{1}{2} \pi\right)\right.$, $R_{\min }=R(\theta=0)$ ). , computations performed using spherical harmonics; $O$, computations performed using oblate spheroidal harmonics.

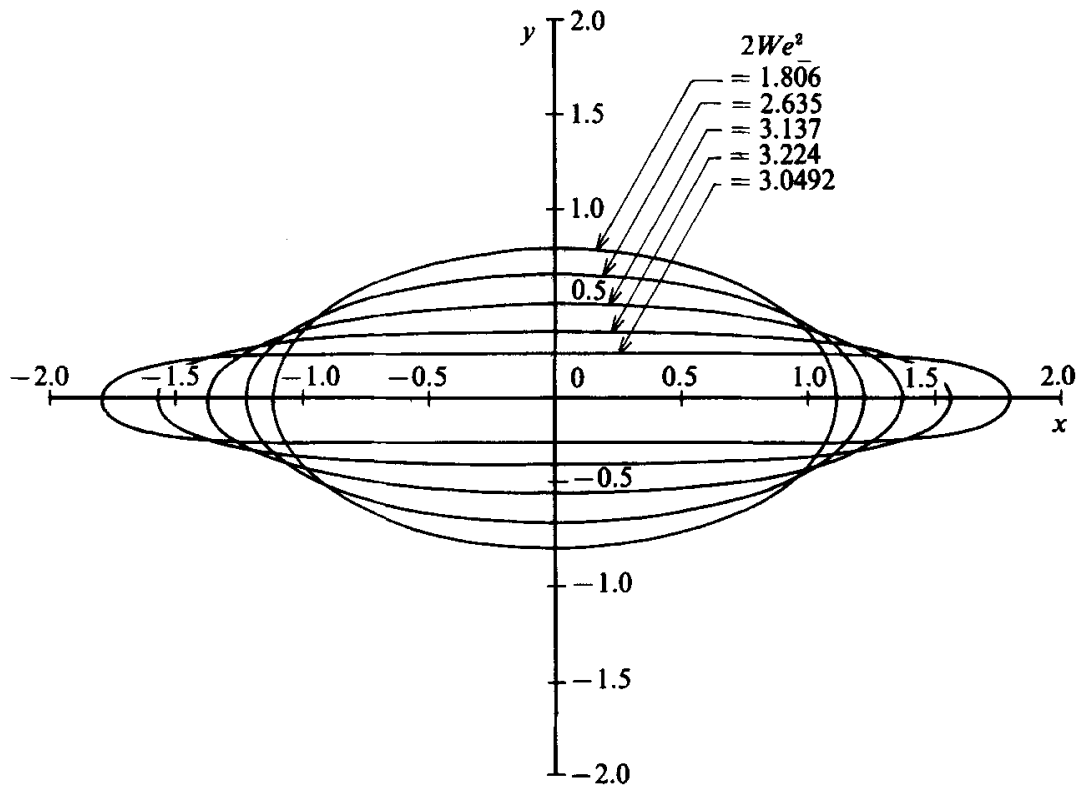

Figure 2. A plot of the axisymmetric bubble shape versus the quantity $\left(2 W e^{2}\right)$. For small values of $W e$ the bubble is almost spherical. For large values the bubble develops negative curvatures about $\theta=0$.

points the convergence of the series representation for the potential was monitored by examining numerically the sequence of partial sums. For all results reported here the series was convergent and we believe our results are accurate to at least five places in all quantities.

In order to compare with previous work we plot in figure 1 the quantity $2 W e^{2}$ versus the aspect ratio of the bubble. It can be seen in agreement with the results of 
Miksis et al. (1981), Moore (1965) and El Sawi (1974) that the Weber number has a broad maximum at $W e=1.27$. To generate solutions near this value it proved convenient to treat the bubble velocity $U$ as an unknown and continue in the oblateness parameter $d$. Past this maximum Weber number the free surface becomes flat near the semiminor axis and the Weber number decreases as the bubble becomes more extended. This effect can be seen in figure 2 where we plot the cross-section of the bubble for various values of the aspect ratio.

Finally, the existence of multiple or non-symmetric solutions due to simple bifurcation was investigated by monitoring the sign of the determinant of the Jacobian as the continuation parameter was varied. As no change of sign was observed for $1 \leqslant R_{\max } / R_{\min } \leqslant 5$ we conclude that there are no simple bifurcations in this range and that if multiple solutions exist they are isolated from the branch we have examined or are associated with bifurcations for which the null space of the singular Jacobian is of even dimension.

\section{Stability}

In this section we examine the stability of the axisymmetric bubble shapes computed in $\$ 2$ to infinitesimal three-dimensional disturbances. The governing equations in three dimensions are (2.2), (2.3). The curvature term for threedimensional surfaces is

$$
\frac{1}{R_{1}}+\frac{1}{R_{2}}=\frac{E D^{\prime \prime}+G D-2 F D^{\prime}}{H^{3}},
$$

where

$$
\begin{gathered}
E=R^{2}+R_{\theta}^{2}, \\
F=R_{\theta} R_{\phi} \\
G=R^{2} \sin ^{2} \theta+R_{\phi}^{2}, \\
H^{2}=R^{2}\left[\sin ^{2} \theta\left(R^{2}+R_{\theta}^{2}\right)+R_{\phi}^{2}\right] \\
D=-R \sin \theta\left(R R_{\theta \theta}-2 R_{\theta}^{2}-R^{2}\right) \\
D^{\prime}=R\left(2 \sin \theta R_{\theta} R_{\phi}-\sin \theta R R_{\theta \phi}+\cos \theta R R_{\phi}\right) \\
D^{\prime \prime}=-R \sin \theta\left(\cos \theta \sin \theta R R_{\theta}+R R_{\phi \phi}-2 R_{\phi}^{2}-\sin ^{2} \theta R^{2}\right) .
\end{gathered}
$$

In order to study the stability of $(2.2),(2.3)$ we let

$$
\left.\begin{array}{rl}
R(\theta, \phi, t) & =\bar{R}(\theta)+\epsilon R^{\prime}(\theta, \phi, t), \\
\Phi(r, \theta, \phi, t) & =\bar{\Phi}(r, \theta)+\epsilon \Phi^{\prime}(r, \theta, \phi, t),
\end{array}\right\}
$$

where $\bar{R}, \bar{\Phi}$ are the steady solutions. Substituting (3.2) into the equations of motion relative to the frame moving with the undisturbed bubble and linearizing in $\epsilon$ we obtain

$$
\begin{gathered}
\nabla^{2} \Phi^{\prime}=0 \\
R_{t}^{\prime}=\Phi_{r}^{\prime}+\bar{\Phi}_{r r} R^{\prime}-\left(\frac{R_{\theta}^{\prime}}{\bar{R}^{2}}-\frac{2 \bar{R}_{\theta} R^{\prime}}{\bar{R}^{3}}\right) \bar{\Phi}_{\theta}-\frac{\bar{R}_{\theta}}{\bar{R}^{2}} \bar{\Phi}_{r \theta} R^{\prime}-\frac{\bar{R}_{\theta}}{\bar{R}^{2}} \Phi^{\prime \theta}, \\
\Phi_{t}^{\prime}=-\left[\left(\bar{\Phi}_{r} \bar{\Phi}_{r r}+\frac{1}{\bar{R}^{2}} \bar{\Phi}_{\theta} \bar{\Phi}_{r \theta}-\frac{2}{\bar{R}^{3}} \bar{\Phi}_{\theta}^{2}\right) R^{\prime}+\bar{\Phi}_{r} \Phi_{r}^{\prime}+\frac{1}{\bar{R}^{2}} \bar{\Phi}_{\theta} \Phi_{\theta}^{\prime}\right]+\frac{\sigma}{\rho} \frac{\delta}{\delta R}\left(\frac{1}{R_{1}}+\frac{1}{R_{2}}\right) R_{1},
\end{gathered}
$$


where $\delta / \delta R$ denotes the functional derivative with respect to $R$. These equations have also been derived in oblate spheroidal coordinates in order to examine the stability of deformed bubbles. Note that both the volume and centroid constraints are no longer enforced in calculating the stability characteristics of the bubble. It is clearly correct to drop the latter as we are interested in deviations of the centroid from a rectilinear path. It is also not necessary to enforce volume conservation provided we limit our considerations to disturbances which initially do not change the volume. The class of disturbances treated by Hartunian \& Sears are of this type. It is necessary to include the equation of state of the gas interior to the bubble if the stability to perturbations which alter the volume is to be examined.

The linear system (3.3)-(3.5) is separable in both the $t$ and $\phi$ variables. It has solutions of the form

$$
\begin{gathered}
R^{\prime}(m, \theta, \phi)=\mathrm{e}^{\omega t} \sum_{l=m}^{\infty} a_{l m} P_{l m}(\theta) \mathrm{e}^{\mathrm{i} m \phi} \\
\Phi^{\prime}(m, r, \theta, \phi)=\mathrm{e}^{\omega t} \sum_{l=m}^{\infty} b_{l m} \frac{P_{l m}(\theta)}{r^{l+1}} \mathrm{e}^{\mathrm{i} m \phi},
\end{gathered}
$$

where the $P_{l m}$ are the associated Legendre polynomials. Note in (3.6)-(3.7) we observe the restriction on the eigenfunctions that for a given azimuthal wavenumber $m, l \geqslant m$. Substituting (3.6)-(3.7) in (3.4)-(3.5) we obtain a generalised eigenvalue problem for the growth rate $\omega$ and the eigenvector $\left\{a_{l m}, b_{l m}\right\}$.

We solve the eigenvalue problem (3.3)-(3.5) by evaluating the equations at the collocation points used to compute the steady solution. Note that there again arises a mismatch in the number of equations and unknowns due to the restriction $l \geqslant m$ in (3.6)-(3.7). If we truncate these series at $l=N$ where $N$ is the number of collocation points used to compute the steady solution we will generate $2 \mathrm{~N}+2$ equations for $2 N+2-2 m$ unknowns. This difficulty may be overcome by truncating the series (3.6)-(3.7) at $l=N+m$. This procedure introduces modes which cannot be properly resolved using $N$ collocation points. The error incurred by this approach will be large for the higher spatial harmonics and thus the eigenvalues which correspond to these harmonics are likely to be spurious. The accuracy of the lower harmonics however should be unaffected. The validity of this procedure was numerically confirmed by monitoring convergence of the eigenvalues as the number of collocation points was increased. The actual eigenvalue problem was solved by the $Q R$ algorithm.

The stability of a stationary gas bubble (i.e. $W e=0$ ) has been examined by Rayleigh. The eigenvalues may be obtained analytically in this limit and are given by (see, for example, Lamb 1932)

$$
\omega^{2}=-(l-1)(l+1)(l+2) \frac{\sigma}{\rho r_{\mathrm{e}}^{3}} .
$$

For values of $l$ greater than 1 it is seen that $\omega^{2}<0$ indicating a stable oscillation of the sphere. Perturbations which alter the volume of the sphere $(l=0)$ are unstable. This instability however only reflects the absence of an equation of state for the bubble interior. Without an equation of state (3.8) is valid only for $l \geqslant 1$. Finally we note that $\omega=0$ for $l=1$. This is a consequence of translational invariance. The shape of the equilibrium solution is independent of the choice of the origin and therefore the equilibrium solution must be exactly neutral $(\omega=0)$ with respect to disturbances which are pure translations of the bubble surface. Eigenfunctions with $l=1$ correspond to translation of the bubble centroid. For $W e>0$ the eigenfunctions will 
no longer be spherical harmonics but translational invariance still implies the existence of a translational mode with $\omega$ identically equal to zero. The above considerations hold however only if the correct steady state is used in performing the stability analysis at a given value of $W e$. We shall demonstrate that if the stability analysis for $W e>0$ is performed with respect to an inconsistent equilibrium solution such as a sphere or an oblate spheroid then translational invariance is violated and a spurious growth rate or oscillation frequency will be obtained for the translational mode.

In order to illustrate this we shall restrict our attention to the effect of infinitesimal translations in the $z$-direction. The invariance of equations (2.6)-(2.7) implies that if $\Phi(r, \theta)$ and $R(\theta)$ are solutions then the one parameter family

$$
\Phi+\epsilon\left(\Phi_{r} \cos \theta-\frac{\sin \theta}{R} \Phi_{\theta}\right), \quad R+\epsilon\left(\cos \theta-\frac{\sin \theta}{R} R_{\theta}\right)
$$

are also solutions. Substituting this family of solutions into the equations of motion and expanding to first order in $\epsilon$ we obtain equations identical to those obtained in the study of the stability of the steady state to small disturbances with the exception that the terms involving the time derivative of the perturbation are absent. This implies that

$$
\Phi^{\prime}=\Phi_{r} \cos \theta-\frac{\sin \theta}{R} \Phi_{\theta}, \quad R^{\prime}=\cos \theta-\frac{\sin \theta}{R} R_{\theta},
$$

are eigenfunctions of the stability problem with eigenvalue zero. We have assumed however that the steady equations were satisfied exactly. In general there will be some error if an approximate steady solution is used. We have then

$$
\begin{gathered}
\Phi_{r}-\frac{R_{\theta}}{R^{2}} \Phi_{\theta}=E_{K}(\theta), \\
\frac{1}{2}\left[\Phi_{r}^{2}+\frac{1}{R^{2}} \Phi_{\theta}^{2}\right]-\frac{\sigma}{\rho}\left[\frac{R^{2}+2 R_{\theta}^{2}-R R_{\theta \theta}+\left(R^{2}+R_{\theta}^{2}\right)\left(1-\left(R_{\theta} / R\right) \cot \theta\right)}{\left(R^{2}+R_{\theta}^{2}\right)^{\frac{3}{2}}}\right] \\
+\frac{p_{\mathrm{i}}}{\rho}=\frac{1}{2 U^{2}}+E_{D}(\theta),
\end{gathered}
$$

where $E_{K}, E_{D}$, are the errors in the kinematic and dynamic conditions respectively.

The relationship between the solution and its derivatives which guarantees the existence of a zero eigenvalue is now violated by an amount comparable to the error in the steady solution. If the errors $E_{K}, E_{D}$ are small then the eigenvalues corresponding to translation will be close to zero. If the errors are large as is the case when a spherical steady state is used in place of the true solution at large values of $W e$, then the eigenvalues can differ considerably from zero. This effect is shown in figure 3. We have followed Hartunian \& Sears and used the spherical approximation.

$$
\bar{R}=r_{\mathrm{e}}, \quad \Phi=U r_{\mathrm{e}}\left[-\frac{r}{r_{\mathrm{e}}} \cos \theta-\frac{1}{2} \frac{P_{1}(\cos \theta) r_{\mathrm{e}}^{2}}{r^{2}}\right],
$$

in (3.4), (3.5). We have plotted the square of the eigenvalue $\left(\left(\lambda^{\prime}\right)^{2}\right.$ in the notation of Hartunian \& Sears) which is zero at $W e=0$. It is seen that $\left(\lambda^{\prime}\right)^{2}$ is initially negative indicating oscillation but eventually changes sign as $W e$ is increased and becomes positive indicating instability. A consistent calculation which takes into account the deformation of the bubble yields the result $\left(\lambda^{\prime}\right)^{2} \equiv 0$. 


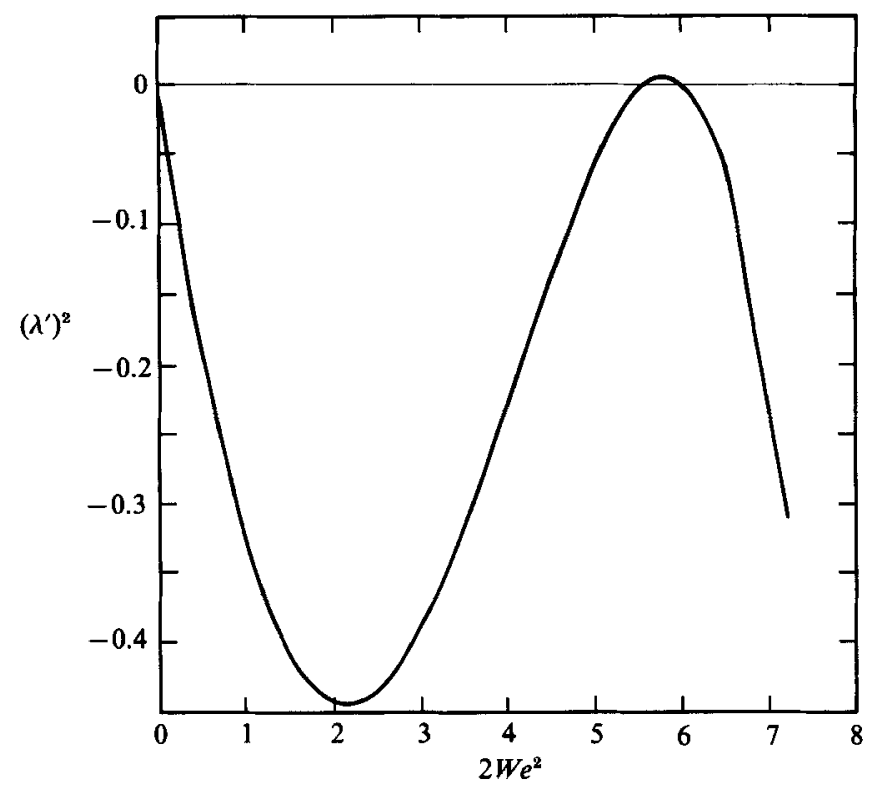

Figure 3. A plot of the square of the eigenvalue corresponding to $l=1, m=1$ as a function of Weber number obtained by using an inconsistent spherical steady state. Note that for $\left(2 W e^{2}=6\right)$ the value of $\left(\lambda^{\prime}\right)^{2}$ is positive indicating a spurious instability. The correct answer, obtained when a consistent steady solution is used, is $\left(\lambda^{\prime}\right)^{2} \equiv 0$.

Since the steady solution is real, the eigenvalues must come in complex conjugate pairs. In addition the equations of motion are invariant under time reversal and it can be shown that if $\omega$ is an eigenvalue, $-\omega$ is also an eigenvalue. In general the eigenvalues must come in quartets if the real and imaginary parts of a given eigenvalue are non-zero. Such a symmetry is in fact a general property of Hamiltonian systems. If $\operatorname{Re}(\omega) \neq 0$ then this corresponds to instability since for every eigenvector with $\operatorname{Re}(\omega)<0$ there exists a corresponding eigenvector with $R e(\omega)>0$. Thus, in contrast with dissipative systems, it is impossible for an inviscid system to have purely decaying eigenfunctions. The notion of spectral stability in an inviscid system implies that all eigenvalues lie on the imaginary axis. Under fairly general assumptions about the smoothness of the steady state solution as $W e$ is varied it can be seen that because of the constraint that the eigenvalues come in quartets the characteristic polynomial for the stability matrix has the property that the transition from a region of spectral stability to one of instability is marked by a coalescence of the imaginary parts of two eigenvalues. This coalescence is a necessary condition for the onset of instability but it is not sufficient. It is possible for a 'near miss' to occur in which two imaginary eigenvalues collide and then move away. Stronger conditions for instability can be found in the work of Mackay \& Saffman (1986) where these ideas are discussed in connection with the stability of water waves.

From (3.8) we see that there is only stable oscillation at $W e=0$. In addition, by virtue of continuity we can label the eigenvectors by the value of $l$ which they take at $W e=0$. Thus we expect the modes to be functions of $l, m$, and the aspect ratio $R_{\max } / R_{\min }$ of the bubble. From the arguments above, instability in such an inviscid system can in fact only occur (at the order of linear theory) if the frequencies at a given value of $m$ but with different values of $l$ coalesce. Coalescence of frequencies 


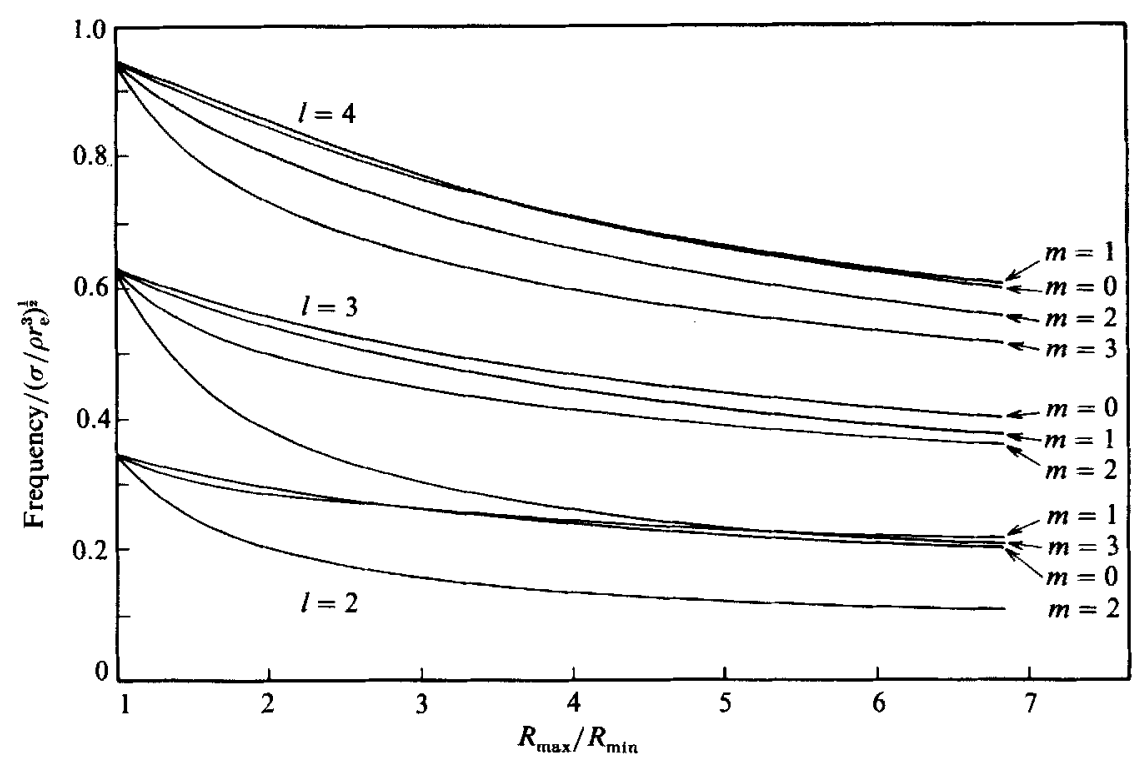

FigURE 4. The frequency of oscillation obtained from linear stability theory as a function of deformation of the bubble. Frequencies are scaled sown by a factor of 10 . Note that the deformation of the bubble breaks the degeneracy of the Rayleigh theory as given in (3.8). Note also that the oscillation frequency is reduced by deformation.

possessing differing values of $m$ is of course also possible but this would not correspond to instability at the level of linear theory. This is due to the fact that our steady state is axisymmetric and the application of separation of variables to the solution of the stability equation shows that the solutions may be partially parametrized by the value of the azimuthal wavenumber $m$.

Convergence of the eigenvalues was monitored by performing the stability analysis with $N=20,40,60$, and 80 collocation points. This confirmed the accuracy of the eigenvalues to at least five places for the range of aspect ratios $1 \leqslant R_{\max } / R_{\min } \leqslant 5$. The results for disturbances with azimuthal wavenumber $m=1,2$ and 3 are shown in figure 4 . It can be seen that the effect of finite Weber number is to reduce the oscillation frequency. Note also that that these disturbances are never unstable for the range of aspect ratios considered. The translational mode labelled $l=1, m=1$ remained zero to five places. Thus our results conflict with those of Hartunian \& Sears who calculated unstable solutions using a sphere and ellipsoid as approximations to the steady state.

\section{Conclusions}

We have developed a numerical technique to study the steady rise and stability of inviscid bubbles. Using this technique we have shown that the conclusions of Hartunian \& Sears on the existence of an inviscid instability are incorrect. Use of a consistent approximation for the bubble steady state reveals no instability in the translation mode (which as shown above is unphysical) nor through a low-order nonlinear resonance mechanism.

We remark that the stability analysis does indicate the existence of spiralling solutions. These solutions are obtained by noting that the form of the solutions to the stability problem developed in $\$ 3$ are in fact travelling waves resulting from the 
restoring force of surface tension. Thus the solution to the stability problem is the first term in an amplitude expansion for these waves. Calculation of higher-order nonlinear corrections results in a finite amplitude solution akin to the well-known solutions in the theory of water waves.

It is easily seen by taking the real and imaginary parts of the complex eigenfunctions calculated in $\$ 3$ that the solutions assume the form of travelling waves. For example, at a finite value of $W e$ the real part of the $(l=2, m=1)$ eigenfunction has the form

$$
\begin{aligned}
& R(\theta, \phi, t)=\bar{R}(\theta)+\epsilon\left[P_{21}(\theta)+\right.\left.a_{41} P_{41}(\theta)+\ldots\right] \cos (\phi+\Omega t) \\
&-\epsilon\left[\alpha_{11} P_{11}(\theta)+a_{31} P_{31}(\theta)+\ldots\right] \sin (\phi+\Omega t), \\
& \Phi(r, \theta, \phi, t)=\bar{\Phi}(r, \theta)+\epsilon\left[b_{21} \frac{P_{21}(\theta)}{r^{3}}+b_{41} \frac{P_{41}(\theta)}{r^{5}}+\ldots\right] \sin (\phi+\Omega t) \\
&-\epsilon\left[b_{11} \frac{P_{11}(\theta)}{r^{2}}+b_{31} \frac{P_{31}(\theta)}{r^{4}}+\ldots\right] \cos (\phi+\Omega t),
\end{aligned}
$$

where $\bar{R}, \bar{\Phi}$ are the axisymmetric steady solutions and $\Omega$ is the angular velocity $(\Omega=\omega / m)$. This form of the solution also suggests that there may exist equilibria of the full three-dimensional equations of motion which represent steady spiralling. Indeed the approximate solution (4.1) represents a bubble whose centroid is displaced from the origin by an infinitesimal amount and thus the path of the centroid is a helix with radius

$$
R_{\mathrm{sp}}=\left[x_{C}^{2}+y_{C}^{2}\right]^{\frac{1}{2}}
$$

and pitch

$$
P_{\mathrm{sp}}=\frac{2 \pi U}{\Omega}
$$

where

$$
\begin{aligned}
& x_{C}=\frac{3}{16 \pi} \int_{0}^{2 \pi} \mathrm{d} \phi \int_{0}^{\pi} \mathrm{d}(\cos \theta) R^{4}(\theta, \phi, t) \sin \theta \cos \phi, \\
& y_{C}=\frac{3}{16 \pi} \int_{0}^{2 \pi} \mathrm{d} \phi \int_{0}^{\pi} \mathrm{d}(\cos \theta) R^{4}(\theta, \phi, t) \sin \theta \sin \phi,
\end{aligned}
$$

and $U$ is the vertical rise velocity.

The eigenfunctions (4.1) represents the first term in an amplitude expansion of solutions of the full three-dimensional equations of motion which are steady in a frame of reference which rotates with angular velocity $\Omega$ and translates with vertical velocity $U$. We emphasize however that the properties of these spiralling solutions even at large values of the amplitude $\epsilon$ are not in accord with experimental observations. The phase speed of these waves $\omega$ is of the order $\left(\sigma / \rho r_{\mathrm{e}}^{3}\right)^{\frac{1}{2}}$. Using estimates for the observed bubble size this yields a frequency which is roughly 10 times the experimentally observed values. Thus these surface tension waves are most likely damped by viscous effects. Limited numerical calculations of the threedimensional equations have shown that finite amplitude effects will not lower appreciably this high rate of spiralling and so details of the calculation are not presented here.

The main conclusion of this work is that inviscid theory does not appear to provide a mechanism for the observed spiralling. We remark that this work only invalidates the use of an irrotational model over the entire bubble. Theories which assume irrotational flow over only the front of the bubble have been developed (Saffman 1956) and their conclusions may remain valid. 
I wish to thank Professor P. G. Saffman for introducing me to this problem. I also wish to acknowledge gratefully many valuable discussions with Professor $\mathrm{D}$. W. Moore and P. G. Saffman.

This work was supported by the Department of Energy, Office of Energy Sciences (DE-AS03-76ER-72012), Applied Mathematical Sciences (KC-07-01-01).

\section{REFERENCES}

Chen, B. \& Saffman, P. G. 1980 Numerical evidence for the existence of new types of gravity waves of permanent form on deep water. Stud. Appl. Maths 62, 1.

Clift, R., Grace, I. R. \& Weber, M. E. 1978 Bubbles, Drops, and Particles. Academic Press.

EL SAwI, M. 1974 Distorted gas bubbles at large Reynolds number. J. Fluid Mech. 62, 163.

Harper, J. F. 1972 Adv. Appl. Mech. 12, 59.

Hartunian, R. A. \& Sears, W. R. 1957 On the stability of small gas bubbles moving uniformly in various liquids. J. Fluid Mech. 3, 27.

LAMB, H. 1945 Hydrodynamics, p. 475. Dover.

Mackay, R. S. \& Saffman, P. G. 1986 Stability of water waves. Proc. R. Soc. Lond. A 406, 115.

Miksis, M., VANDEN-Broeok, J. \& Keller, J. B. 1981 Axisymmetric bubble or drop in a uniform flow. J. Fluid Mech. 108, 89.

Miksis, M., VANDen-Broeck, J. \& Keller, J. B. 1982 Rising bụbbles. J. Fluid Mech. 123, 31.

MOORE, D. W. 1965 The velocity of rise of distorted gas bubbles in a liquid of small viscosity. J. Fluid Mech. 23, 749.

Rosenberg, B. 1950 The drag and shape of air bubbles moving in liquids. David Taylor Model Basin Rep. 727.

Ryskin, G. \& Leal, L. G. $1984 a$ Numerical solution of free boundary problems in fluid mechanics. Part 1. The finite difference technique. J. Fluid Mech. 148, 1.

Ryskin, G. \& LEaL, L. G. $1984 b$ Numerical solution of free boundary problems in fluid mechanics. Part 2. Buoyancy-driven motion of a gas bubble through a quiescent liquid. J. Fluid Mech. 148, 19.

Saffman P. G. 1956 On the rise of small air bubbles in water. J. Fluid Mech. 1, 249. 